\title{
Dosimetric Evaluation of Advanced Radiotherapy Techniques in Treating Patients with Prostatic Cancer
}

\author{
Amin E. Amin ${ }^{1}$, Somaia M. El Sayed ${ }^{1}$, Ahmed Ezzat ${ }^{2}$ \\ ${ }^{1}$ Medical Physics, Radiation Oncology and Nuclear Medicine Department, Faculty of Medicine, Ain \\ Shams University, Cairo, Egypt; ${ }^{2}$ Clinical Oncology, Clinical Oncology and Nuclear Medicine \\ Department, Faculty of Medicine, Ain Shams University, Cairo, Egypt
}

Background: Different radiotherapy techniques were developed to deliver optimal dose to prostate cancer while sparing nearby organs at risk (OARs).

Aim: To compare different dosimetric methods used to evaluate sparing of OARs and to select radiotherapy plan which provides the most OARs sparing during prostate cancer treatment.

Methods: We used computed tomography data sets of 10 patients. For each data set, six plans were calculated; two RapidArc, two intensity-modulated radiation therapy (IMRT), and two 3-dimensional conformal radiotherapy (3DCRT) plans. Isodose distribution and dose volume histograms (DVHs) were used for plan analysis and evaluation. The dose to OAR was compared by calculating the DVH for each OAR in different plans. The dose (D) delivered to certain percentage (n) of an organ (Dn) was then determined. In addition, the Critical Organ Scoring Index (COSI) and the modified COSI (mCOSI) were calculated.

Results: 3DCRT with 5 fields produced the lowest rectal dose, RapidArc with bladder avoidance sector (RapidArcBladder) produced the lowest bladder dose and IMRT with 9 beams produced the lowest dose to the femoral heads. The COSI and MCOSI values of the two IMRT plans were significantly lower than those of 3DCRT and RapidArc (<0.0001). The calculation of mCOSI produced similar results to that obtained with COSI $(<0.0001)$.

Conclusion: The results of this study showed that COSI and MCOSI are better dosimetric methods in selecting the plan with more sparing of OARs than Dn. Meanwhile among advanced radiotherapy techniques, RapidArc and 3DCRT are more sparing of OARs than IMRT.

Keywords: Radiotherapy planning, Organ at risk, Dosimetric evaluation, Critical Organ Scoring Index (COSI) Corresponding author: Ahmed Ezzat, MD; Clinical Oncology Department, Faculty of Medicine, Ain Shams University, Cairo, Egypt; E-mail: Ahmedezzat200011@gmail.com

Submitted: 15-September-2017, Revised: 19-October-2017, Accepted: 23-October-2017, Published online: 1-November-2017

\section{INTRODUCTION}

Prostate cancer incidence is growing all over the world due to the rise in the median age of population and the increasing prostate-specific antigen screening. Prostate cancer is the $6^{\text {th }}$ most common type of cancer worldwide and the $2^{\text {nd }}$ most predominant in men, accounting for about $10 \%$ of all cancer cases ${ }^{1}$.

The optimum treatment of localized prostate cancer stays debatable. There are three main options of treatment; surgery, radiotherapy (external beam radiotherapy or brachytherapy) and hormonal therapy ${ }^{2}$. Radiation therapy is one of the main lines of treatment in early, locally advanced and even metastatic prostate cancer. In the new era of advanced radiotherapy techniques such as intensity-modulated radiation therapy (IMRT), several trials have documented its excellent results especially in the treatment of prostate cancer. IMRT permits dose escalation with high conformity and high dose to the prostate and while sparing normal tissues such as the bladder and rectum ${ }^{2-4}$. Moreover, IMRT results were much better as regards toxicity in comparison to three-dimensional conformal radiotherapy (3DCRT) technique plans ${ }^{5-7}$. Therefore, IMRT is being increasingly used in the treatment of prostate cancer.
Various modalities of IMRT delivery systems have been developed, such as linear accelerator based-IMRT systems, the Tomotherapy® system (Hi-Art system, Accuray Inc., Madison, WI, USA), the RapidArc system (Varian Medical System Inc., Palo Alto, CA, USA), the volumetric modulated arc therapy (VMAT) system (Elekta Instrument AB Stockholm), and the CyberKnife system (Accuray Inc., Sunnyvale, CA, USA), and proton systems.

Dosimetric studies have compared treatment plans using different IMRT modalities in patients with prostate cancer ${ }^{8-12}$. The dosimetric outcome differed when comparing plans using RapidArc and the VMAT in comparison to IMRT in the treatment of prostate cancer 13-24. Many studies observed better normal tissue sparing with VMAT over IMRT when including simple target volume (prostate with or without seminal vesicles) though shortened treatment time did not differ between both modalities 12, 14, 16, 17, 19-21, 23. However, very few studies have focused on more complex pelvic target volumes, containing the prostate, seminal vesicles and pelvic lymph nodes 15, 18, 22, 24 . Some of these studies found largely equivalent sparing of organs at risk (OARs) between VMAT and IMRT ${ }^{8}$, 14. In contrast, other studies have reported contradictory results, 
showing IMRT technique to give superior OARs sparing in comparison to VMAT ${ }^{15}$. Myrehaug et al found VMAT to have no consistent dosimetric advantage over IMRT ${ }^{22}$. Thus, those studies have produced diverse results.

The aim of this study was to examine a dosimetric evaluation method to evaluate sparing of OARs in advanced radiotherapy techniques. Then, we apply the evaluation method to select radiotherapy plan which provides most OARs sparing in prostate cancer.

\section{METHODS}

\section{Cases and Plans}

In this study, we used computed tomography data sets from 10 patients who had been previously treated with 3DCRT for prostate cancer at the Clinical Oncology Department, Ain Shams University Hospitals.

For designing treatment plans and calculating dose distributions for all applied radiotherapy techniques, Eclipse treatment planning system V13.5 (Varian
Medical System Inc., Palo Alto, CA, USA) with AAA algorithm have been used.

For each data set, six plans have been calculated; two RapidArc, two IMRT, and two 3DCRT plans. The planning target volume (PTV) was contoured by expanding the prostate contour with a $10 \mathrm{~mm}$ margin in all directions except in the posterior direction where only $8 \mathrm{~mm}$ margin was applied to spare additional rectal tissue from receiving radiation dose. The prescribed dose was $76 \mathrm{~Gy}$ in 38 fractions over 52 days. The dose was prescribed and normalized at the isocenter point in all techniques.

Figure 1 shows the different plans studied in this work. The studied plans were as follows:

- 3DCRT: Two different plans with different number and arrangements of fields:

- 3DCRT with five fields (3DCRT-5): the beam angles of this plan were $0^{\circ}, 50^{\circ}, 90^{\circ}$, $270^{\circ}$ and $310^{\circ}$ (Figure 1.A).

- 3DCRT with seven fields (3DCRT-7): the beam angles of this plan were $0^{\circ}, 50^{\circ}, 90^{\circ}$, $130^{\circ}, 220^{\circ}, 270^{\circ}$ and $310^{\circ}$ (Figure 1.B).

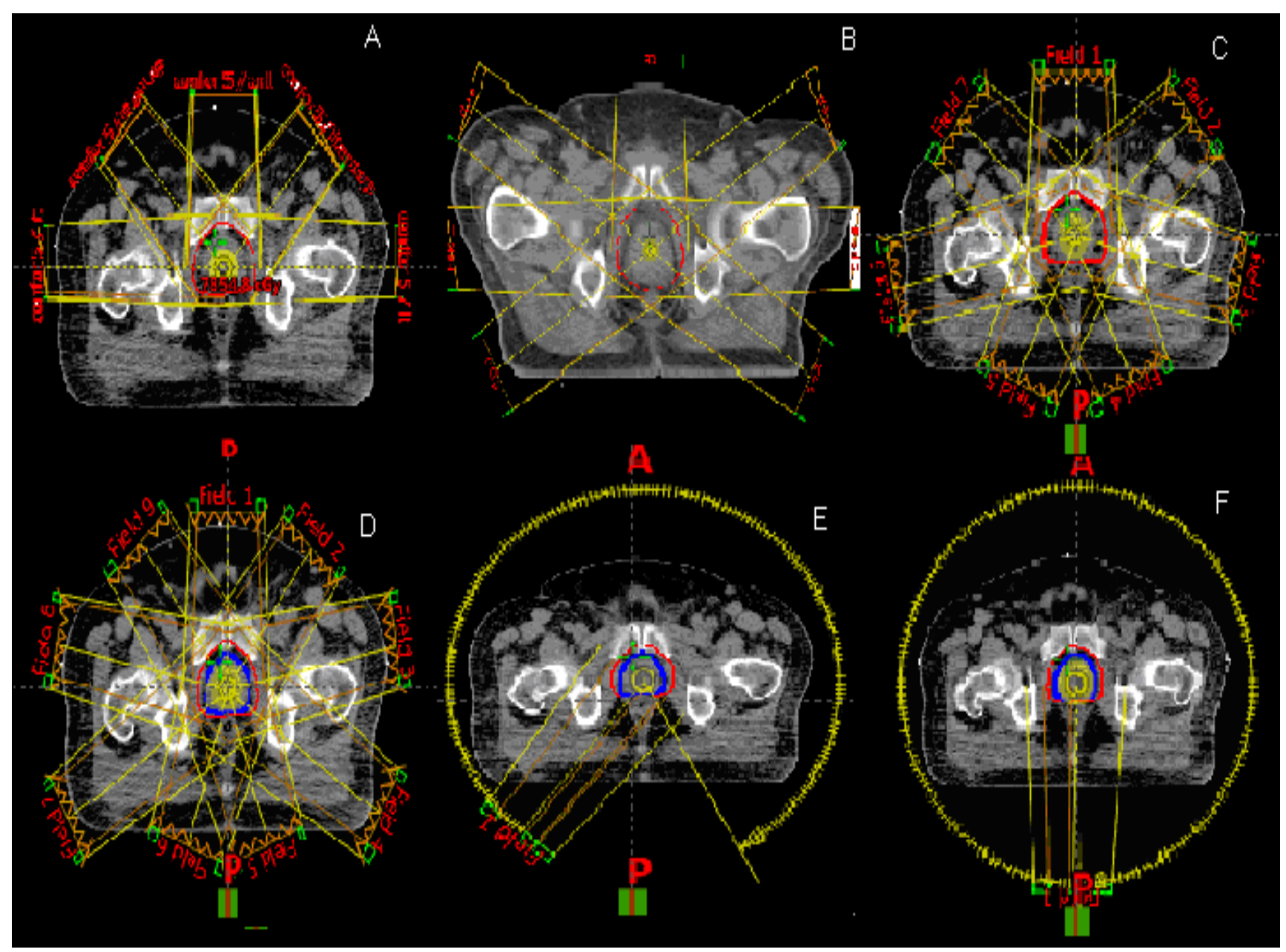

Figure 1: Beam arrangement for six plans. A: 3DCRT with five fields (3DCRT-5); B: 3DCRT with seven fields (3DCRT-

7); C: IMRT with seven fields (IMRT-7); D: IMRT with nine fields (IMRT-9); E: Single arc with $300^{\circ}$ arc angle (RapidArc-rectum); F: A single full arc (RapidArc-bladder). 
- IMRT: Two different plans with different number and arrangements of fields:

- IMRT with seven fields (IMRT-7): The beams were arranged with equal angle separation of 51 degrees between each two beams. IMRT-7 had gantry angles of $0^{\circ}$, $51^{\circ}, 102^{\circ}, 153^{\circ}, 204^{\circ}, 255^{\circ}$ and $306^{\circ}$ (Figure 1.C).

- IMRT with nine fields (IMRT-9): The beams were arranged with equal angle separation of 40 degrees between each two beams. IMRT- 9 had gantry angle of $0^{\circ}$, $40^{\circ}, 80^{\circ}, 120^{\circ}, 160^{\circ}, 200^{\circ}, 240^{\circ}, 280^{\circ}$, and $320^{\circ}$ (Figure 1.D).

- RapidArc: Two different plans with different arc shapes:

- RapidArc-Rectum: A single arc with $300^{\circ}$ arc that started with a gantry angle of $150^{\circ}$ and rotated in a counter clockwise to gantry angle $210^{\circ}$. The arc deliberately avoided treating through the rectum from the posterior direction (Figure 1E).

- RapidArc-Bladder: A single full arc utilized an arc that started with the gantry at $179^{\circ}$ and rotated in a counter clockwise to gantry angle $181^{\circ}$, for a total $360^{\circ}$ arc. An anterior $60^{\circ}$ avoidance sector was applied to avoid the bladder anteriorly. The avoidance sector started at $330^{\circ}$ to $30^{\circ}$. In the RapidArc plans, the collimator was set at $30^{\circ}$ to minimize the multileaf collimator tongue and groove effect (figure $1 \mathrm{~F}$ ).

\section{Data analysis}

Both of isodose distribution and dose volume histograms (DVHs) were used for plan analysis and evaluation. DVHs were calculated and generated based on 3D reconstructed images for PTV and all OARs in treatment plans. A dose distribution was considered acceptable for treatment if it is able to meet the prescribed prostate planning dose-volume constrains outlined in table 1 . The target coverage was quantitatively assessed by using dosimetrical indexes like prescription isodose to target volume ratio, homogeneity index, conformity index (CI), target coverage index (TCI), conformity number $(\mathrm{CN})$ and dose gradient.

Isodose distribution and DVH analysis were insufficient to distinguish which plan was superior in terms of higher sparing of OARs. As a result, more dosimetric calculations have been performed for each OAR. The dose to the OAR was compared by calculating the DVH for each OAR in different plans. Then, determining the dose delivered to certain percentage of an organ (Dn). For the rectum and the bladder; D15, D25, D35 and D50 were determined. For the head of femur; D0 (Dmax), D25 and D40 were determined. In addition to that, two more indexes were calculated to account for the irradiation of all OARs regarding their tolerance. One of these indexes is the Critical Organ Scoring Index (COSI) which takes into account both the target coverage and the critical organ irradiation ${ }^{25,26}$. The main advantage of this index is its ability to account of the irradiation of all OARs simultaneously in one index. The COSI can be expressed as:

$$
\mathrm{COSI}=1-\sum_{1}^{n} w_{i} \frac{V_{i}(O A R)_{>t o l}}{T C}
$$

Where " $V(\mathrm{OAR})_{>\text {tol }}$ " is the fraction of the volume of OAR that receives more than a predefined tolerance dose, and TC is the volumetric target coverage, which is defined as the fractional volume of the PTV covered by the prescribed isodose. The target coverage (TC) is defined as the ratio of the target volume receiving at least the prescription dose $\left(V_{\mathrm{T}, \mathrm{PI}}\right)$ to the total target volume $\left(V_{\mathrm{T}}\right)$. Typically; the coverage index should be at least $95 \%$. TC is expressed as: $\mathrm{TC}=V_{\mathrm{T}, \mathrm{PI}} / V_{\mathrm{T}}{ }^{25}$.

COSI may also be expressed as:

$$
\text { COSI }=1-\frac{V(\mathrm{OAR})_{>\text {tol }}}{\mathrm{TC}_{V}}
$$

In this study we used the first equation for calculating COSI. The reason of using the first equation is accounting for the irradiation of the four OARs simultaneously.

The other index that was calculated in this study is the modified COSI (mCOSI) which is expressed as:

$$
\operatorname{mCOSI}=\sum_{i=1}^{n} W i\left[\frac{\operatorname{Cos} I 10+\operatorname{Cos} I 20+\cdots+\operatorname{Cos} I 80}{8}\right]
$$

Although the COSI focuses only on OARs receiving high dose region volumes, the mCOSI considers both high dose and low dose regions.

Table 1: Planning objectives for intensity modulated

\begin{tabular}{|c|c|}
\hline $\begin{array}{l}\text { Volume/ } \\
\text { OAR }\end{array}$ & Dose constraint \\
\hline \multirow[t]{4}{*}{ PTV } & $\begin{array}{l}99 \% \text { of the volume to get } \geq 95 \% \text { of the } \\
\text { prescription }\end{array}$ \\
\hline & Minimum dose $>90 \%$ of the prescription \\
\hline & Maximum dose $<107 \%$ of the prescription \\
\hline & The maximum dose must be within the PTV \\
\hline \multirow[t]{4}{*}{ Rectum } & $<50 \%$ of the volume to receive $60 \mathrm{~Gy}$ \\
\hline & $<35 \%$ of the volume to receive $65 \mathrm{~Gy}$ \\
\hline & $<25 \%$ of the volume to receive $70 \mathrm{~Gy}$ \\
\hline & $<15 \%$ of the volume to receive $75 \mathrm{~Gy}$ \\
\hline \multirow[t]{4}{*}{ Bladder } & $<50 \%$ of the volume to receive $65 \mathrm{~Gy}$ \\
\hline & $<35 \%$ of the volume to receive $70 \mathrm{~Gy}$ \\
\hline & $<25 \%$ of the volume to receive $75 \mathrm{~Gy}$ \\
\hline & $<15 \%$ of the volume to receive $80 \mathrm{~Gy}$ \\
\hline \multirow{3}{*}{$\begin{array}{l}\text { Head of } \\
\text { femur }\end{array}$} & $<45 \%$ of the volume to receive $40 \mathrm{~Gy}$ \\
\hline & $<25 \%$ of the volume to receive $45 \mathrm{~Gy}$ \\
\hline & $0 \%$ of the volume to receive $50 \mathrm{~Gy}$ \\
\hline
\end{tabular}
radiation therapy (IMRT) and volumetric-modulated arc therapy (RapidArc) treatments of the prostate 
Statistical analysis was conducted using Graphpad Prism version 7 for windows (www.graphpad.com). Statistical comparison between the evaluation factors of different plans was done using the one way ANOVA test. To be statistically different, the values were needed to be significant at the $95 \%$ level (i.e. $\mathrm{p}<0.05$ ).

\section{RESULTS}

Doses delivered to OARs are presented in table 2. 3DCRT-5 produced the lowest rectal dose. The rectal doses in the other five plans were very close. However, the differences of the four dosimetric doses of the rectum between all plans were not statistically significant. The RapidArc-bladder produced the lowest bladder dose which was statistically significant when compared to the other techniques. The bladder doses in the other five plans were very close. The differences in the four dosimetric doses of the bladder in all other plans were not statistically significant. IMRT-9 produced statistically significant lower doses to the left and right femoral heads.

The COSI and mCOSI values of all applied plans are shown in table 2. The COSI values of the 3DCRT and RapidArc plans were very close with no significant difference. The COSI values of the two IMRT plans were lower than that of 3DCRT and RapidArc and the difference between them was statistically significant. This means that 3DCRT and RapidArc may spare all OARs simultaneously more than IMRT.

Calculation of the mCOSI produced similar results to that obtained with COSI. The mCOSI values of both 3DCRT and RapidArc were very close with no significant difference. The mCOSI values for the IMRT plans were significantly lower than those of 3DCRT and RapidArc plans. This confirms that 3DCRT and RapidArc may spare all OARs simultaneously more than IMRT.

\section{DISCUSSION}

Radiation treatment planning analyses usually depend on DVH as the most widely used dosemetric tool to physically evaluate different plans. The results of this study indicated that the selection of a plan depending on dose volume objectives only may spare one OAR but not the others. Accordingly, we examined two other methods, the COSI and the mCOSI to evaluate dosesparing of all OARs simultaneously in different treatment plans for prostate cancer.

Table 2: Doses to organs at risk using the six radiotherapy techniques

\begin{tabular}{|c|c|c|c|c|c|c|c|c|}
\hline \multirow{3}{*}{ OAR } & \multicolumn{6}{|c|}{ Radiotherapy technique } & \multirow[t]{3}{*}{$\mathbf{F}$} & \multirow[t]{3}{*}{ p-value } \\
\hline & 3DCRT-5 & 3DCRT-7 & IMRT-7 & IMRT-9 & $\begin{array}{l}\text { RapidArc - } \\
\text { rectum }\end{array}$ & $\begin{array}{l}\text { RapidArc - } \\
\text { bladder }\end{array}$ & & \\
\hline & \multicolumn{6}{|c|}{ Mean \pm SD } & & \\
\hline \multicolumn{9}{|l|}{ Rectum } \\
\hline D50\% & $38 \pm 7.76$ & $35 \pm 7.41$ & $43 \pm 8.78$ & $42 \pm 8.34$ & $37 \pm 7.62$ & $34 \pm 6.27$ & 2.1897 & 0.0687 \\
\hline D35\% & $47 \pm 9.93$ & $49 \pm 8.43$ & $56 \pm 9.90$ & $55 \pm 9.59$ & $48 \pm 8.68$ & $51 \pm 8.13$ & 1.6764 & 0.1561 \\
\hline D25\% & $51.6 \pm 8.96$ & $55 \pm 8.88$ & $62 \pm 9.13$ & $61 \pm 9.33$ & $54 \pm 8.15$ & $60 \pm 9.24$ & 2.2869 & 0.06 \\
\hline D15\% & $59 \pm 8.83$ & $65 \pm 8.27$ & $70 \pm 10.1$ & $69 \pm 9.87$ & $62 \pm 8.67$ & $67 \pm 9.50$ & 2.0970 & 0.0798 \\
\hline \multicolumn{9}{|l|}{ Bladder } \\
\hline D50\% & $27 \pm 4.5$ & $26 \pm 4.1$ & $31 \pm 5.8$ & $30 \pm 3.9$ & $27 \pm 4.3$ & $17 \pm 2.1$ & 13.6017 & $<0.0001$ \\
\hline D35\% & $34 \pm 4.5$ & $32 \pm 4.8$ & $37 \pm 5.1$ & $35 \pm 5.4$ & $33 \pm 4.2$ & $24 \pm 2.3$ & 10.0338 & $<0.0001$ \\
\hline $\mathrm{D} 25 \%$ & $41 \pm 5.8$ & $40 \pm 6.1$ & $45 \pm 5.6$ & $46 \pm 4.9$ & $43 \pm 5.2$ & $32 \pm 3.1$ & 9.3449 & $<0.0001$ \\
\hline $\mathrm{D} 15 \%$ & $51 \pm 5.9$ & $49 \pm 5.1$ & $53 \pm 5.6$ & $52 \pm 5.3$ & $50 \pm 4.8$ & $42 \pm 4.1$ & 5.808 & $<0.0001$ \\
\hline \multicolumn{9}{|l|}{ Lt. Femur } \\
\hline $\mathrm{D} \max \%$ & $50 \pm 9.3$ & $39 \pm 7.2$ & $42 \pm 7.6$ & $29 \pm 2.8$ & $48 \pm 7.2$ & $47 \pm 6.9$ & 11.3455 & $<0.001$ \\
\hline D 25\% & $29 \pm 4.3$ & $31 \pm 3.3$ & $27 \pm 2.3$ & $22 \pm 2.3$ & $28 \pm 3.3$ & $30 \pm 3.5$ & 9.6672 & $<0.001$ \\
\hline $\mathrm{D} 40 \%$ & $30 \pm 5.1$ & $31 \pm 5.3$ & $26 \pm 2.1$ & $20 \pm 1.3$ & $27 \pm 3.3$ & $28 \pm 4.2$ & 10.2784 & $<0.001$ \\
\hline \multicolumn{9}{|l|}{ Rt. Femur } \\
\hline Dmax\% & $42 \pm 6.7$ & $39 \pm 4.6$ & $40 \pm 4.8$ & $29 \pm 5.3$ & $41 \pm 4.5$ & $40 \pm 5.4$ & 8.1758 & $<0.0001$ \\
\hline D25\% & $31 \pm 4.9$ & $30 \pm 5.2$ & $32 \pm 4.3$ & $22 \pm 1.6$ & $34 \pm 3.5$ & $33 \pm 3.8$ & 11.3372 & $<0.0001$ \\
\hline D40\% & $33 \pm 6.1$ & $32 \pm 5.6$ & $28 \pm 3.5$ & $21 \pm 2.1$ & $31 \pm 3.3$ & $30 \pm 4.8$ & 9.5502 & $<0.0001$ \\
\hline$\overline{\text { COSI }}$ & $\begin{array}{l}0.828 \pm \\
0.10\end{array}$ & $\begin{array}{l}0.817 \pm \\
0.11\end{array}$ & $\begin{array}{l}0.615 \pm \\
0.041\end{array}$ & $\begin{array}{l}0.610 \pm \\
0.062\end{array}$ & $\begin{array}{l}0.835 \pm \\
0.12\end{array}$ & $\begin{array}{l}0.843 \pm \\
0.15\end{array}$ & 11.8815 & $<0.0001$ \\
\hline mCOSI & $\begin{array}{l}0.126 \pm \\
0.021\end{array}$ & $\begin{array}{l}0.116 \pm \\
0.021\end{array}$ & $\begin{array}{l}0.075 \pm \\
0.010\end{array}$ & $\begin{array}{l}0.056 \pm \\
0.011\end{array}$ & $\begin{array}{l}0.114 \pm \\
0.031\end{array}$ & $\begin{array}{l}0.109 \pm \\
0.032\end{array}$ & 14.6399 & $<0.0001$ \\
\hline
\end{tabular}

OAR: Organ at risk; 3DCRT: three-dimensional conformal radiotherapy; IMRT: intensity-modulated radiation therapy; COSI: Critical Organ Scoring Index; mCOSI: modified Critical Organ Scoring Index 
Another advantage of COSI and mCOSI is the evaluation of TC as well as evaluating the sparing of OARs. Our results showed that using a certain technique may spare one OAR but not the other which may be spared by another technique. On the other hand, COSI and mCOSI may specify the technique with the most dose-sparing of all OARs.

The COSI was described by Menhel et al in 2006 as a new index to compare between radiotherapy plans ${ }^{25}$. They tested the new index on the planning of the following sites: head and neck, cavernous sinus and pancreas. They compared treatment plans of conformal noncoplanar 3D and IMRT techniques. For all three sites the usage of COSI index was so helpful in comparing between the treatment plans and facilitated the choice of the optimal one. Analysis of individual DVHs and isodose lines were done and the results were compared to the widely used conformation number. They confirmed the efficiency of COSI in assessing different treatment plans through evaluating the different dosimetric parameters and choosing the optimal plan. The COSI tool was found to be objective, quick and accurate tool in choosing the best plan. Similar to our results, Menhel et al found that a non-coplanar 3D plan was superior to the IMRT plan ${ }^{25}$.

Levin et al in their retrospective study on ten patients with maxillary sinus tumors compared treatment plans of 9-field IMRT, directionally optimized IMRT, and 3D noncoplanar planning in terms of critical organ sparing and TC ${ }^{27}$. They used the COSI in the analysis of different treatment plans and compared it to other widely used conformation numbers. In cases where the choice of best treatment plan was difficult, the 2D COSI-CI index gave an accurate evaluation of $\mathrm{TC}$ and OAR overdoses, even in the situation where other conformity indices failed. Both IMRT techniques gave comparable results. They delivered lower doses to ipsilateral structures while 3D plans delivered lower doses to contralateral structures with more homogeneous dose distribution.

In this study, we compared the quality of RapidArc technique with that of IMRT and 3DCRT in the treatment of prostate cancer patients with prophylactic pelvic radiotherapy and simultaneous dose escalation to the prostate. All the three techniques were able to meet all of our plan acceptance criteria. The results showed that RapidArc and 3DCRT resulted in slightly superior conformity of prostate PTV as they have higher COSI and mCOSI than IMRT. The COSI and mCOSI values indicated also that RapidArc was more sparing of OARs (bladder, rectum and heads of femur) than IMRT. These results are in compliance with RapidArc demonstrating better conformity to the PTV than IMRT. Similar results were observed by Elith et al in their study on twenty prostate cancer cases ${ }^{28}$. They assessed the plan quality of both VMAT and IMRT and concluded that IMRT plans had the advantages of more homogeneous dose distribution and less time in calculation, while the VMAT plans gave better conformity to the target volume with more sparing of OAR.

Lee et al performed a planning study on ten patients with prostate cancer ${ }^{29}$. Different modalities of IMRT were used, like the linac step and shoot, RapidArc and proton systems. Analysis and comparison between different treatment plans were carried out using variable dosimetric indexes such as COSI, homogeneity index, quality factor, and conformation number. Superior conformity index, homogeneity index, and $\mathrm{CN}$ values in addition to higher COSI were recorded with RapidArc plan denoting better coverage of PTV. In terms of more sparing of OAR especially in high dose volumes, the tomotherapy and RapidArc plans were much better than other techniques.

Our observation is different from that reported by Yoo et al ${ }^{15}$. They reported that IMRT plans in cases with large PTVs (including the prostate, seminal vesicles and lymph nodes) were more sparing to the bladder, rectum, and small when compared to the RapidArc technique. Meanwhile for small PTVs (including the prostate and seminal vesicles) both conventional IMRT and RapidArc technique with two arcs were comparable as regards OAR sparing. The differences in their results could be explained as they increased the number of beams for IMRT, which shows better results with more beams in general. The differences in the volume and shape may be also contributing. Another factor is the dose-volume constraints for OARs and their weightings as they tightened the dose-volume constraints during the optimization process. Also the treatment planning using RapidArc was still in its early stages, whereas treatment planning using IMRT has actively progressed over 10 years.

\section{Conclusion}

The results of the current study shows that COSI and mCOSI are better in selecting the plan with more sparing of OARs than Dn. When comparing the advanced radiation therapy modalities, RapidArc and 3DCRT were found to be more sparing of OARs than IMRT in the treatment of patients with prostate cancer.

\section{REFERENCES}

1. Torre LA, Bray F, Siegel RL, Ferlay J, Lortet-Tieulent J, Jemal A. Global cancer statistics, 2012. CA Cancer J Clin. 2015; 65(2): 87-108

2. Intensity Modulated Radiation Therapy Collaborative Working Group. Intensity-modulated radiotherapy: current status and issues of interest. Int $\mathbf{J}$ Radiat Oncol Biol Phys. 2001; 51(4): 880-914.

3. Luxton G, Hancock SL, Boyer AL. Dosimetry and radiobiologic model comparison of IMRT and 3D conformal radiotherapy in treatment of carcinoma of the prostate. Int J Radiat Oncol Biol Phys. 2004; 59(1): 267284.

4. Vlachaki MT, Teslow TN, Amosson C, Uy NW, Ahmad S. IMRT versus conventional 3DCRT on prostate and normal tissue dosimetry using an endorectal balloon for prostate immobilization. Med Dosim. 2005; 30(2): 69-75.

5. De Meerleer G, Vakaet L, Meersschout S, Villeirs G, Verbaeys A, Oosterlinck W, De Neve W. Intensitymodulated radiotherapy as primary treatment for prostate cancer: acute toxicity in 114 patients. Int J Radiat Oncol Biol Phys. 2004; 60: 777-787.

6. Sethi A, Mohideen N, Leybovich L and Mulhall J. Role of IMRT in reducing penile doses in dose escalation for 
prostate cancer. Int J Radiat Oncol Biol Phys. 2003; 55(4): 970-978.

7. Wang L, Hoban P, Paskalev K, et al. Dosimetric advantage and clinical implication of a micro-multileaf collimator in the treatment of prostate with intensitymodulated radiotherapy Med Dosim. 2005; 30(2), 97-103.

8. Ceylan C, Kucuk N, Bas Ayata H, Guden M and Engin K. Dosimetric and physical comparison of IMRT and CyberKnife plans in the treatment of localized prostate cancer. Rep Pract Oncol Radiother. 2010; 15(6): 181-189.

9. Cotter SE, Herrup DA, Friedmann A, et al. proton radiotherapy for pediatric bladder/prostate rhabdomyosarcoma: clinical outcomes and dosimetry compared to intensity-modulated radiation therapy. Int $\mathbf{J}$ Radiat Oncol Biol Phys. 2011; 81(5): 1367-1373.

10. Schwarz M, Pierelli A, Fiorino $C$, et al. Helical tomotherapy and intensity modulated proton therapy in the treatment of early stage prostate cancer: a treatment planning comparison. Radiother Oncol. 2011; 98(1): 7480.

11. Amin AE, El Sayed AM, El Sayed EM, Sallam AM, Talaat MS. Evaluation of modern radiotherapy techniques in treatment of cancer prostate. Int J Sci Technol. 2016; 4(6): 122-139.

12. Amin AE, Hamed NA, El Sayed EM, Sallam AM, El Sayed AM. Physical evaluation and verification of different radiotherapy technique for prostate cancer Int $\mathbf{J}$ Sci Technol. 2017; 5(5); 58-70.

13. Palma D, Vollans E, James $\mathrm{K}$, et al. Volumetric modulated arc therapy for delivery of prostate radiotherapy: comparison with intensity-modulated radiotherapy and three dimensional conformal radiotherapy. Int J Radiat Oncol Biol Phys. 2008; 72(4): 996-1001.

14. Kjaer-Kristoffersen F, Ohlhues L, Medin J, Korreman S. RapidArc volumetric modulated therapy planning for prostate cancer patients. Acta Oncol. 2009; 48(2): 227232.

15. Yoo S, Wu J, Lee R, Yin FF. Radiotherapy treatment plans with rapidarc for prostate cancer involving seminal vesicles and lymph nodes. Int J Radiat Oncol Biol Phys. 2010; 76(3): 935-942.

16. Zhang P, Happersett L, Hunt M, Jackson A, Zelefsky M, Mageras G. Volumetric modulated arc therapy: planning and evaluation for prostate cancer cases. Int $\mathrm{J}$ Radiat Oncol Biol Phys. 2010; 76(5): 1456-1462.

17. Aznar MC, Petersen PM, Logadottir A, et al. Rotational radiotherapy for prostate cancer in clinical practice. Radiother Oncol. 2010; 97(3):480-484.

18. Davidson MT, Blake SJ, Batchelar DL, Cheung P, Mah $\mathrm{K}$. Assessing the role of volumetric modulated arc therapy (VMAT) relative to IMRT and helical tomotherapy in the management of localized, locally advanced, and post- operative prostate cancer. Int J Radiat Oncol Biol Phys. 2011; 80(5): 1550-1558

19. Hardcastle N, Tome WA, Foo K, Miller A, Carolan M, Metcalfe P. Comparison of prostate IMRT and VMAT biologically optimized treatment plans. Med Dosim. 2011; 36(3): 292-298.

20. Kopp RW, Duff M, Catalfamo F, Shah D, Rajecki M, Ahmad K. VMAT vs. 7-field- IMRT: assessing the dosimetric parameters of prostate cancer treatment with a 292-patient sample. Med Dosim. 2011; 36(4): 365-372.

21. Sale C, Moloney P. Dose comparisons for conformal, IMRT and VMAT prostate plans. J Med Imaging Radiat Oncol. 2011; 55(6): 611-621.

22. Myerhaug S, Chan G, Craig T, et al. A treatment planning and acute toxicity comparison of two pelvic nodal volume delineation techniques and delivery comparison of intensity-modulated radiotherapy versus volumetric modulated arctherapy for hypofractionated high-risk prostate cancer radiotherapy. Int $\mathbf{J}$ Radiat Oncol Biol Phys. 2012; 82(4): e657-662.

23. Sze HC, Lee MC, Hung WM, Yau TK, Lee AW. RapidArc radiotherapy planning for prostate cancer: single-arc and double-arc techniques vs. intensitymodulated radiotherapy. Med Dosim. 2012; 37(1): 87-91.

24. Fontenot JD, King ML, Johnson SA, Wood CG, Price MJ, Lo KK. Single-arc volumetric modulated Arc therapy can provide dose distributions equivalent to fixed-beam intensity-modulated radiation therapy for prostatic irradiation with seminal vesicle and/or lymph node involvement. Br J Radiol. 2012; 85(1011): 231-236.

25. Menhel J, Levin D, Alezra D, Symon Z, Pfeffer R. Assessing the quality of conformal treatment planning: a new tool for quantitative comparison. Phys Med Biol. 2006; 51(20): 5363-5375.

26. Pyakuryal A, Myint WK, Gopalakrishnan M, Jang S, Logemann JA, Mittal BB. A computational tool for the efficient analysis of dose-volume histograms for radiation therapy treatment plans. J Appl Clin Medl Phys. 2010; 11(1): 3013.

27. Levin D, Menhel J, Alezra D, Pfeffer R. IMRT vs. 3D noncoplanar treatment plans for maxillary sinus tumors: a new tool for quantitative evaluation. Med Dosim. 2008; 33(4): 275-281.

28. Elith CA, Dempsey SE, Warren-Forward HM. A retrospective planning analysis comparing intensitymodulated radiation therapy (IMRT) to volumetricmodulated arc therapy (VMAT) using two optimization algorithms for the treatment of prostate cancer. J Med Radiat Sci. 2013; 60(3): 84-92.

29. Lee S, Cao YJ, Chang KH, et al. Treatment plan comparison of linac step and shoot, tomotherapy, rapidarc, and proton therapy for prostate cancer by using the dosimetrical and the biological indices. J Korean Phys Soc. 2015; 67(1): 7-16. 